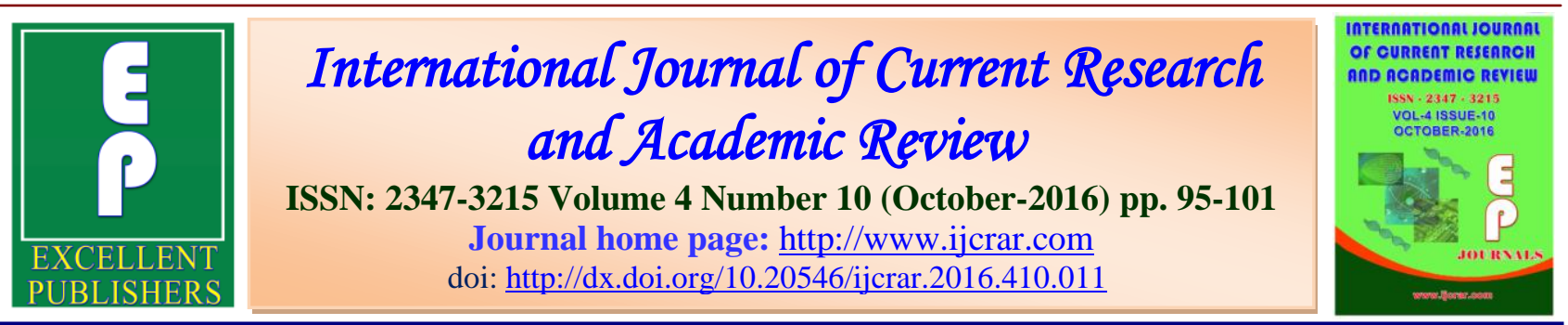

\title{
A Comparative Study of Serum Levels of HE4 and CA125 in Women with Ovarian Masses
}

\author{
Fatemeh Cheraghi $^{1}$, Zeinab Omidi ${ }^{2}$ and Farideh Moramezi ${ }^{3}$ \\ ${ }^{1}$ Department of Obstetrics and Gynecology, Fertility Infertility and Perinatology Research \\ Center, Imam Khomeini Hospital, Ahvaz Jundishapur University of Medical Sciences, Ahvaz, \\ Iran \\ ${ }^{2}$ Student Research Committee, Ahvaz Jundishapur University of Medical Sciences, Ahvaz, \\ Iran \\ ${ }^{3}$ Fertility, Infertility and Perinatology Research Center, Imam Khomeini Hospital, Medicine \\ Faculty, Ahvaz Jundishapur University of Medical Sciences, Ahvaz, IR Iran \\ *Corresponding author
}

\section{KEYWORDS \\ Cancer, ovarian cancer, HE-4,} CA125.

\section{A B S T R A C T}

Currently there is no appropriate clinical algorithm to prioritize and triage patients with malignant tumors to the medical centers for surgery. Studies have shown that HE4 alone has higher specificity than CA125 in diagnosis of ovarian tumors and the combination of these two tumor markers has higher accuracy in detecting malignancies than each one alone. The aim of this study was to find a tumor marker that could be used alone or in combination with other markers for the early detection of ovarian cancers especially the epithelial type as well as their differentiation from benign masses and therefore appropriate triage of patients with malignant masses to medical equipped centers for surgery. In this descriptive - retrospective study 86 patients with ovarian masses hospitalized in Ahwaz Imam Khomeini Hospital for surgery were studied. The records of the patients who had been hospitalized within the years of 2011-2013 was analyzed and the HE4 and CA125 serum levels were measured by ELISA method and also the information in the patient records were recorded in demographic questionnaire and compared. SPSS version 18 was used for statistical analysis. Levels of both markers CA125 and HE4 were significantly higher in epithelial cases $(\mathrm{P}=0.0001)$. CA125 marker frequency distribution based on benign or malignant tumor was not significantly different in patients under study $(\mathrm{P}>0.05)$ but the marker HE4 was significantly different based on the type of tumor (benign or malignant) in patients under study and its level was higher in malignant cases $(\mathrm{P}=0.002)$. Based on the results obtained in the study it is concluded that HE4 is a better marker with better efficiency for the differentiation of benign and malignant ovarian tumors and also in malignant cases it is applied in epithelial and Germ cell differentiation but it has no use in the diagnosis of sex cord tumor and germ cell and thus it is recommended to apply it in patients with a diagnosis of ovarian tumors. 


\section{Introduction}

One of the problems in today's healthcare is cancer that is dramatically increasing in the world (Rahmani et al., 2016; Firoozabadi et al., 2015; Mohammad et al., 2015). Investigating the causes of death among women has shown that ovarian cancers are the second leading cause of death and among the reproductive system cancers ovarian cancer is the fourth leading cause of death in women (Bowtell et al., 2015). In Arab et al the rate of ovarian cancer in Iran is reported as 2.3 per hundred thousand women in 2005-2004 (Arab et al., 2010). The average age of developing ovarian cancer in Iran is 46.7 for the epithelial ovarian tumors and the age of 34.3 has been reported for the non-epithelial tumors (Khodabakhshi et al., 2007). Almost $90 \%$ of ovarian cancers are associated with epithelial ovarian cancer that is heterogeneous and it is classified based on the type of cell that is involved that includes serous, mucinous, endometrial, clear cell and Brenner and it matches with different types of ovarian epithelial cells in female reproductive system. These tumors are classified into three groups: benign, borderline and malignant. Mucinous and endometrial tumors are usually malignant and invasive carcinoma. But serous tumors are not usually invasive.

In $75 \%$ of cases due to late diagnosis of the illness, in spite of extensive surgery and chemotherapy, patients' survival is poor and patients with high-stage ovarian cancer are treated with surgery and chemotherapy but despite great advances in the field of cancer, only $5 \%$ of women with stage IV of the disease survive for 5 years and thus the early diagnosis is very critical (Lurie et al., 2007; Horne et al., 2014). It should be noted that the use of specific tumor markers that have the diagnosis power in the early stages is an extremely effective action in improving health outcomes. It should be noted that currently there is no is no appropriate clinical algorithm to prioritize and triage patients with malignant tumors to the medical centers for surgery. That is why different cancer markers are introduced for screening and diagnosis of cancer types that CA125 (carcinoembryonic antigen 125) is one of the oldest of these markers and used to predict the risk of malignancy in a pelvic mass but there are shortcomings in the field of working with these cancer markers including the low level of specificity and sensitivity of this marker in many benign diseases in women as well as many other malignancies such as lung cancer and lymphoma (Lu et al., 2004). One of the new markers of ovarian cancer is human epididymis protein 4 or HE4. This marker is discovered by expression rows RNA in normal and malignant ovarian epithelial cells. Studies have shown that HE4 alone has higher specificity than CA125 in diagnosis of ovarian tumors and the combination of these two tumor markers has higher accuracy in detecting malignancies than each one alone. The aim of this study was to find a tumor marker that could be used alone or in combination with other markers for the early detection of ovarian cancers especially the epithelial type as well as their differentiation from benign masses and therefore appropriate triage of patients with malignant masses to medical equipped centers for surgery.

\section{Materials and Methods}

After obtaining permission from Ethics Committee of the University, a descriptive retrospective study was conducted on 86 patients with ovarian masses hospitalized in Ahwaz Imam Khomeini Hospital for surgery. The records of the patients who had been hospitalized within the years of 20112013 was analyzed and the HE4 and CA125 serum levels were measured by ELISA 
method and also the information in the patient records were recorded in demographic questionnaire and compared. The cut-off levels of $\mathrm{He} 4$ were considered as $70 \mathrm{Pmol} / \mathrm{Lit}$ and200 U/mL.

After collecting the required data using SPSS version 18 software the collected data was analyzed and for the qualitative variables the frequency and frequency percentage and for quantitative variables the mean and standard deviations were calculated. In order to evaluate hypotheses the independent t-test and ANOVA were used and the level of significance for the interpretation of the results was considered as 0.05 .

\section{Results and Discussion}

In this study, 86 patients with ovarian tumors were studied. The average age distribution based on the tumor in patients shows that the benign masses include $46.51 \%$ and malignant masses include $53.48 \%$ of the total samples under study. The average age in benign cases was 45.31 and 43.28 in malignant cases. Epithelial tumors include $72 \%$, germ cell tumors form
$22 \%$ and sex cord tumors (granulosa cell tumor) include $6 \%$ of the total samples. The amount of both markers CA125 (619.41 + 898.98 versus $105.73+87.45)$ was significantly higher in cases of epithelial ( $\mathrm{P}$ $=0.0001)$. The level of CA125 marker was significantly higher in Sex Cord patients $(233.98+104.58$ versus $491.01+819.18)$. The level of HE4 was significantly lower in Sex Cord patients $(50.98+24.13$ versus 220.66+293.93) ( $\mathrm{P}=0.0001)$. The level of CA125 was significantly lower in Germ Cell groups that the rest of cases $(71.99+39.64$ versus $590.65+870.64$ and $\mathrm{P}=0.0001)$. Also the level of HE4 was significantly lower in Germ Cell groups that the rest of cases $(39.93+20.01$ versus $259.25+309.69$ and $P$ $=0.0001)$. The frequency distribution of the marker CA125 based on the benign or malignant tumor had no significant difference in patients $(403.07+579.01$ versus $539.53+949.45 \mathrm{P}>0.05)$, but the marker HE4 based on the type of tumor (benign or malignant) had a significant difference in patients and its value was higher in malignant cases $(111.85+368.59$ versus $296.84+72.255 \mathrm{P}=0.002)$.

Table.1 Age frequency distribution based on tumor type in patients under study

\begin{tabular}{|c|c|c|c|c|c|}
\hline Tumor & Total & Pathology & No & $\%$ & Age \\
\hline \multirow{4}{*}{ Benign } & \multirow{4}{*}{40} & Dermoid & 12 & $13 / 95$ & $(60-18) 39 / 45$ \\
\hline & & Serous cyst adenoma & 15 & $17 / 44$ & $(61-19) 42 / 4$ \\
\hline & & Mucinous cyst adenoma & 11 & $12 / 79$ & $(62-22) 45 / 4$ \\
\hline & & Adeno-fibroma cyst & 2 & 2/32 & $(50-28) 54$ \\
\hline \multirow{7}{*}{ Malignant } & \multirow{7}{*}{46} & Immature Tratoma & 2 & 2/32 & $(58-45) 51 / 5$ \\
\hline & & Dysgerminoma & 2 & 2/32 & $(58-14) 36$ \\
\hline & & Yolk Sac Tumor & 3 & $3 / 48$ & $(41-17) 29 / 3$ \\
\hline & & Granulosa tumor & 5 & $5 / 81$ & $(60-43) 51 / 4$ \\
\hline & & Serous cyst carcinoma & 17 & 19/76 & $(82-24) 47 / 9$ \\
\hline & & Mucinous cyst carcinoma & 15 & $17 / 44$ & $(68-20) 44 / 4$ \\
\hline & & Endometrioid tumor & 2 & $2 / 32$ & $(50-30) 42 / 5$ \\
\hline
\end{tabular}


Table.2 CA125 and HE4 Level in Patient sort by epithelial status, Sex cord status, Germ cell status and Type

\begin{tabular}{|c|c|c|c|c|c|c|}
\hline \multirow[t]{2}{*}{ variable } & \multirow{2}{*}{$\begin{array}{l}\text { Sub- } \\
\text { Variable }\end{array}$} & \multicolumn{2}{|l|}{ Ca125 } & \multicolumn{2}{|l|}{ HE4 } & \multirow{2}{*}{$\begin{array}{l}\text { P- } \\
\text { Value }\end{array}$} \\
\hline & & Mean & SD & Mean & SD & \\
\hline \multirow[t]{2}{*}{ Epithelial } & Positive & 619.41 & 898.98 & 276.05 & 316.06 & \multirow[t]{2}{*}{0.0001} \\
\hline & Negative & 105.73 & 87.45 & 42.23 & 20.87 & \\
\hline \multirow[t]{2}{*}{ Sex cord } & Positive & 233.98 & 104.58 & 50.98 & 24.13 & \multirow[t]{2}{*}{0.0001} \\
\hline & Negative & 491.01 & 819.18 & 220.66 & 293.93 & \\
\hline \multirow{2}{*}{$\begin{array}{l}\text { Germ } \\
\text { cell }\end{array}$} & Positive & 71.99 & 39.64 & 39.93 & 20.01 & \multirow[t]{2}{*}{0.0001} \\
\hline & Negative & 590.65 & 870.64 & 259.25 & 309.69 & \\
\hline \multirow[t]{2}{*}{ Type } & Benign & 403.07 & 579.01 & 111.85 & 72.255 & \multirow[t]{2}{*}{0.002} \\
\hline & Malignant & 539.53 & 949.45 & 296.84 & 368.59 & \\
\hline
\end{tabular}

HE4 is a secreted protein the expression of which is increased in certain cancers thus this marker could play an important role in the diagnosis and follow up in these patients. Studies have particularly shown that this marker has diagnosis ability alone and can diagnose with high sensitivity in combination with CA125 (Kondalsamy et al., 2013; O'Shannessy et al., 2013). As previously stated the aim of this study was to find a tumor marker that could be used alone or in combination with other markers for the early detection of ovarian cancers especially the epithelial type as well as their differentiation from benign masses and therefore appropriate triage of patients with malignant masses to medical equipped centers for surgery. In this study it became clear that out of 86 patients with ovarian tumors 40 cases $(46.51 \%)$ were associated with benign masses and 46 cases $(53.48 \%)$ were associated with malignant masses that the benign masses contained 12 cases of mature teratoma $(13.95 \%), 15$ cases of serous cyst adenoma (17.44\%), 11 cases of mucinous cyst adenoma (12.79\%) and 2 cases of adeno-fibroma cyst $(2.23 \%)$ and malignant masses contained 2 cases of immature teratoma $(2.32 \%), 2$ cases of dysgerminoma (2.32\%), 3 cases of Yolk Sac Tumor $(3.48 \%), 5$ cases of granulosa tumor
(5.81\%), 17 cases of serous cyst carcinoma $(19.76 \%), 15$ cases of mucinous cyst carcinoma $(17.44 \%)$ and 2 cases of endometrioid tumor $(2.32 \%)$. The results obtained in this study showed that the value of both markers was significantly higher in epithelial cases and it was significantly lower in Germ cell cases; but there was no significant difference between Sex cord and the rest of tumors. Meanwhile, the frequency distribution of marker CA125 based on the type of tumor was not significantly different in patients but the marker HE4 was significantly different in terms of tumor type and its value was higher in malignant cases.

Ghasemi et al., (2014) in Yazd concluded that in order to predict the advanced stage of the disease the combination of HE4 and CA125 was more appropriate to predict cancer but this was not investigated in the present study. A cohort study in 2013-2010 in China by Wen-Ting Chen et al., showed that HE4 serum level was closely related to surgical outcome. Of course the present study was cross-sectional and patients' prognosis was not analyzed. Grigorios Kalapotharakos et al., (2012) in Sweden concluded that the high plasma levels of HE4 was a marker independent of poor prognosis in non-ovarian cancers that is not consistent with the results of the present 
study. Cristina Anton \& Partners (2011) compared the diagnostic accuracy of CA125 and HE4 in ovarian masses and concluded that there was no difference between the accuracy of CA125 and HE4 to differentiate types of ovarian tumors that is not consistent with the results of the present study. Rafael Molina et al., (2011) in Spain by comparing HE4 with CA125 concluded that HE4 alone could be used for ovarian tumor diagnosis and has higher specificity than CA125 and it is consistent with the results of the present study. In Hala A Abdel-Azeez et al., (2010) it was concluded that HE4 has the highest sensitivity in ovarian tumor diagnosis especially in the early stages of the disease which confirms the results of the present study. Richard G. Moore et al., (2007) concluded that HE4 alone has the highest sensitivity in ovarian tumor diagnosis, which is consistent with the results of the present study. Valsive et al., in the USA observed that increased level of CA-125 alone is not sufficient to differentiate benign and malignant pelvic masses that the results of the present study also indicate the same result. Rustin et al., (2000) in a cohort study stated that increasing CA-125 to more than 30 units per $\mathrm{ml}$ is associated with the disease progression and recurrence of epithelial ovarian cancer which is not discussed in the present study. Kim et al., conducted a cohort study and in South Korea that its results were published in 2008 and it was concluded that by measuring CA-125 level it is possible to predict the results of surgery and chemotherapy in patients with epithelial ovarian cancer which can be addressed in future studies of the Iranian patients as well.

In general based on the results of this study it can be concluded that HE4 is a better marker with higher efficiency to differentiate the benign and malignant ovarian tumors and also is useful in malignant cases in the differentiation of epithelial and Germ cell types but it cannot be applied in sex cord tumor and germ cell differentiation and thus it is recommended to apply it in patients with a diagnosis of ovarian tumors. In the end it is proposed to conduct the future multi-center studies with higher sample volume and to confirm these results.

\section{References}

Arab, M., et al. 2010. Incidence rate of ovarian cancer in Iran in comparison with developed countries. Indian J. Cancer, 47: 322-327.

Bowtell, D.D., Böhm, S., Ahmed, A.A., Aspuria, P.J., Bast, Jr. R.C., Beral, V., Berek, J.S., Birrer, M.J., Blagden, S., Bookman, M.A., Brenton, J.D. 2015. Rethinking ovarian cancer II: reducing mortality from high-grade serous ovarian cancer. Nature Reviews Cancer, 15(11): 668-79.

Cristina Anton, et al. 2012. A comparison of CA125, HE4, risk ovarian malignancy algorithm (ROMA), and risk malignancy index (RMI) for the classification of ovarian masses. Obstetrics and Gynecol., 67(5):437441.

Firoozabadi, M.D., Rahmani, H. 2015. Prevention of nausea and vomiting: methods and utility after surgery in cancer patients? Asian Pacific J. Cancer Prevention, 16(7): 2629-35.

Grigorios Kalapotharakos, et al. High preoperative blood levels of HE4 predicts poor prognosis in patients with ovarian cancer. J. Ovarian Research, 5.(20)

Hala, A., Abdel-Azeez, et al. HE4 and Mesothelin: Novel Biomarkers of Ovarian Carcinoma in Patients with Pelvic Masses. Asian Pacific Journal of Cancer Prevention, 2010; 11: 111116. 
Horne, H.N,. Sherman, M.E., Garcia-Closas, M., Pharoah, P.D., Blows, F.M., Yang, X.R., et al. 2014. Breast cancer susceptibility risk associations and heterogeneity by E-cadherin tumor tissue expression. Breast Cancer Res. Treat., 143(1): 181-7.

Jonathan, S., Berek, 2002. Gynecol., Vol. 2, 1065-1129.

Khodabakhshi, R., et al. 2007. Treatment and prognosis of epithelial ovarian cancer in Iran. J. Clin. Oncol., 25(18): 5586.

Kim, H.S., Park NH, Chung HH, Kim JW, Song YS, Kang SB. Significance of preoperative serum CA-125 levels in the prediction of lymph node metastasis in epithelial ovarian cancer. Acta Obstet Gynecol Scand. 2008;87(11):1136-42.

Kondalsamy- Chennakesavan, S., Hackethal, A., Bowtell, D., Obermair, A. 2013. Australian Ovarian Cancer Study Group. Differentiating stage 1 epithelial ovarian cancer from benign ovarian tumours using a combination of tumour markers HE4, CA125, and CEA and patient's age. Gynecologic Oncol., 129(3): 467-71.

Lu, K.H., et al. 2004. Selection of potential markers for epithelial ovarian cancer with geneexpression arrays and recursive descent partition analysis. Clin. Cancer Res., 10(10): 3291-300.

Lurie, G., Wilkens, L.R., Thompson, P.J., McDuffie, K.E., Carney, M.E., Terada, K.Y., et al. 2007. Vitamin D receptor gene polymorphisms and epithelial ovarian cancer risk. Cancer Epidemiol. Biomarkers Prev.,16(12): 2566-71.

Meyer, T., Rustin, G.J. Role of tumour markers in monitoring epithelial ovarian cancer. $\mathrm{Br} . \mathrm{J}$. Cancer. 2000;82:1535-1538.

Mohammad Ali Sheikhi, Ahmad Ebadi, Abdolhassan Talaeizadeh, and Hossein
Rahmani. 2015. "Alternative Methods to Treat Nausea and Vomiting from Cancer Chemotherapy," Chemother. Res. Practice, vol. 2015, Article ID $818759, \quad 6 \quad$ pages, 2015. doi:10.1155/2015/818759

Nolen, B., et al. 2010. Serum biomarker panels for the discrimination of benign from malignant cases in patients with an adnexal mass. Gynecol. Oncol., 117: 440-445.

O’Shannessy, Daniel, J., et al. 2013. "Serum folate receptor alpha, mesothelin and megakaryocyte potentiating factor in ovarian cancer: association to disease stage and grade and comparison to CA125 and HE4." J. Ovarian Res., 6.1: 1.

Ortiz-Muñoz, B., Aznar-Oroval, E., García. AG, Peris AC, Ballestero PP, Yepes MS, Lozano TG, Ballester CI, Garcia EG. HE4, Ca125 and ROMA algorithm for differential diagnosis between benign gynaecological diseases and ovarian cancer. Tumor Biology. 2014 Jul 1;35(7):7249-58.

Rafael Molina, et al. 2011. HE4 a novel tumour marker for ovarian cancer: comparison with CA 125 and ROMA algorithm in patients with gynaecological diseases. Tumor. Biol., 32: 1087-1095.

Rahmani, H., Shahriary, A., Sheikhi, M.A., Ebadi, A., Davoodzadeh, H. 2016. Applications of cardio-toxicity in breast cancer: a meta-analysis. Panminerva medica.

Ren, J, et al. Tumor markers for early detection of ovarian cancer. Expert Rev. Mol. Diagn., 2010; Sep;10(6):787-98.

Richard, G. Moore, et al. The use of multiple novel tumor biomarkers for the detection of ovarian carcinoma in patients with a pelvic mass. 
Int.J.Curr.Res.Aca.Rev.2016; 4(10): 95-101

Gynecologic Oncology. 2008; 108:402-408.

Richard, G., Moore. et al. 2008. The use of multiple novel tumor biomarkers for the detection ofovarian carcinoma in patients with a pelvic mass. Gynecologic Oncol., 108: 402-408.

Seidman, J.D., R.P., Kurman, R.J. 2002. Surface epithelial tumors of the B.s.P.o.t.F.G. Tract. New York, 2002: 791-904.
Valsive, Steven, A., M.D., Schlaerth John, B. M.D. et al. 1988. Serum levels in preoperative evaluation of pelvic masses. Am. J. Obstet. Gynecol., 73(4): 266-70.

Wen-Ting Chen, et al. 2014. HE4 as a Serum Biomarker for ROMA Prediction and Prognosis of Epithelial Ovarian Cancer Asian Pacific $J$. Cancer Prevention, 15(1): 101-105.

\section{How to cite this article:}

Fatemeh Cheraghi, Zeinab Omidi and Farideh Moramezi. 2016. A Comparative Study of Serum Levels of HE4 and CA125 in Women with Ovarian Masses. Int.J.Curr.Res.Aca.Rev.4(10): 95-101. doi: http://dx.doi.org/10.20546/ijcrar.2016.410.011 